\title{
Concepciones sobre las matemáticas, su enseñanza y su aprendizaje de docentes en formación ${ }^{1}$
}

\author{
Conceptions about mathematics, its teaching \\ and learning of teachers in training \\ Concepções sobre a matemática, seu ensino e \\ aprendizagem de professores em formação
}

Recibido: mayo 2013

Aceptado: agosto 2013

Willington Algeri Benítez Chará

\section{Resumen}

El trabajo que se presenta forma parte de una investigación llevada a cabo con docentes en ejercicio y docentes en formación, de la Facultad de Educación de la Universidad del Cauca, con la finalidad de identificar sus Concepciones sobre las Matemáticas, su Enseñanza y Aprendizaje (Benítez; 2011), a través de la realización de entrevistas semiestructuradas.

Palabras clave: Enseñanza; metodología de enseñanza; análisis y reflexión sobre la enseñanza; concepciones sobre matemáticas; enseñanza y aprendizaje de las matemáticas; profesor; formación de profesores.

\begin{abstract}
The work presented is part of a research conducted with practicing teachers and student teachers, Faculty of Education, University of Cauca, in order to identify their conceptions about mathematics, his Teaching and Learning (Benitez , 2011), through semi-structured interviews.
\end{abstract}

Keywords: Other notions of Mathematics Education; systems of representation, semiotics processing; school math, algebra, polynomials remarkable product.

\section{Resumo}

O trabalho apresentado é parte de uma pesquisa realizada com professores praticantes e professores de estudantes da Faculdade de Educação da Universidade de Cauca, a fim de identificar suas concepções sobre a matemática, seu ensino e aprendizagem (Benitez , 2011), através de entrevistas semi-estruturadas.

\footnotetext{
Articulo de Investigación

Licenciado en Educación con especialidad en Matemáticas, Especialista en Educación Matemática, Magister en Educación por la Universidad del Cauca. Docente Institución Educativa Santa Rosa- Docente cátedra Universidad del Cauca- Docente cátedra Institución Universitaria Colegio Mayor del Cauca-. Grupo de Educación Matemáticas Universidad del CaucaGEMAT. Contacto: willingtonbenitez@gmail.com
} 
Palavras-chave: educação, ensino metodologia, análise e reflexão sobre o ensino, as concepções de matemática de ensino e aprendizagem da matemática, professor, formação de professores.

\section{Introducción}

En el campo del estudio de las matemáticas, entendido como el conjunto de actividades, personas e instituciones dedicadas a la producción y comunicación del conocimiento matemático, se considera que los profesores y los estudiantes mantienen unas concepciones acerca de los conceptos matemáticos y del aprendizaje y la enseñanza de los mismos. En este orden de ideas, se afirma que las concepciones de los profesores de matemáticas inciden en la toma de decisiones y en su forma de actuar al interior del aula de matemáticas, razón por la cual, dentro de una amplia gama de tópicos de interés para la investigación sobre las concepciones del profesor, se hace necesario indagar por las concepciones que tienen los profesores las Matemáticas, su enseñanza y aprendizaje.

Investigaciones como las de Ernest (1989), Thompson (1992); Flores (1998), etc., han mostrado que lo que un profesor cree sobre la enseñanza y el aprendizaje de las matemáticas y lo que conoce del contenido, métodos y materiales disponibles para enseñar matemáticas influyen en sus decisiones relativas a la enseñanza. En este sentido estas concepciones son la base en la que se deben fundamentar las investigaciones que pretendan comprender las decisiones que los docentes toman.

En particular las concepciones se van formando a lo largo de la vida del docente, desde su formación inicial como estudiante donde se empieza a consolidar su práctica, hasta llegar a arraigarse progresivamente en su rol como de docente en ejercicio. y son más estables cuanto más tiempo llevan formando parte de sus sistemas de creencias. Por ello, se constituyen en una especie de lente o de filtro que los estudiantes utilizan, consciente o inconscientemente, para filtrar los contenidos de la Didáctica de las Matemáticas de los cursos de formación e interpretar su propio proceso formativo.
Esto lo lleva a una adaptación de sus estructuras y propicia en él una visión particular acerca de las matemáticas, la cual trata de trasmitir a sus alumnos a través de la enseñanza en el aula, recurriendo, a veces, a diferentes tipos de enseñanza y aprendizaje que le permite enfatizar en aspectos distintos de las matemáticas. Los docentes en formación, muchas de las concepciones son implícitas, por lo que durante sus cursos universitarios debieran reflexionar sobre ellas y hacerlas explícitas, aunque, la reflexión sobre sus concepciones no garantiza de forma automática su transferencia a la práctica del aula. El sistema de creencias, le dan al profesor la capacidad de cambiar sus concepciones $\mathrm{y}$, como consecuencia, sus prácticas.

Se infiere de lo anterior la existencia de una relación entre la enseñanza de las matemáticas y alguna de las concepciones sobre el las matemáticas. Al respecto, Thom (1973, citado en: Ponte, 1999) afirma que: "Detrás de cualquier modelo de enseñanza de las matemáticas hay una filosofía de las matemáticas". En este sentido, uno de los aspectos que nos parece importante es hacer reflexionar a los docentes en formación acerca de sus propias concepciones sobre las matemáticas, su enseñanza y aprendizaje, como uno de esos recursos que podemos utilizar para mejorar su desempeño en el aula. A este respecto, se busca identificar las concepciones sobre las matemáticas, su enseñanza y aprendizaje de los docentes en formación, del Programa de Licenciatura en matemáticas, de la Facultad de Educación de la Universidad del Cauca

\section{Metodología}

Procurando dar cuenta del alcance pretendido, se determinó como método el enfoque de investigación cualitativo, y de corte interpretativo, en la medida en que se aspira a entender los modos de ver la realidad de los informantes; y transversal, en 
cuanto que seleccionamos 6 y 4 informantes, respectivamente de cada grupo de docentes, con una característica común. Para el estudio se realizó una entrevista semiestructurada en profundidad cuya finalidad es identificar y realizar las situaciones más sensibles y particulares de sus experiencias con las matemáticas de cada uno de los sujetos en la investigación, así como los argumentos que esgrime para justificar sus posiciones y sus prácticas (Remesal, 2006).

Sujetos participantes. La población objeto de estudio estuvo conformada por los seis docentes en ejercicio y cuatro docentes en formación de los tres últimos semestres del programa de Licenciatura Facultad de Ciencias Naturales, Exactas y de la Educación de la Universidad del Cauca

\section{Resultados}

Al tratar de dar respuesta a los diferentes interrogantes planteados por Benítez (2011), entre los que se encuentra ¿cómo aprenden sus estudiantes las matemáticas?, son diversas LAS posiciones que adoptan los docentes en formación

El estudiante es el protagonista en su proceso de formación y el principal interesado en aprovechar bien los años de universidad. Como cualquier persona en situación similar, está muy influenciado por las formas de ser y hacer de sus profesores y, en general, por el ambiente de la institución universitaria y el grupo social en el que se integra, cada estudiante está muy condicionado por una multitud de influencias: su temperamento, sus experiencias previas, sus estados de ánimo, los grupos sociales en los que se integra, etc. influyen en su ilusión personal y sus proyectos de futuro; en su estilo de aprendizaje, etc.

Ante la pregunta propuesta, a los futuros maestros ¿Cómo aprenden las matemáticas?; sus respuestas se centraron básicamente en los siguientes elementos, a partir de una buena lectura de los conceptos matemáticos y la realización de un sin número de ejercicios, mediante la cual el estudiante va adquiriendo destrezas y dominio de los mismos, así como también plantean que las matemáticas se aprenden a través de la reflexión permanente, la retroalimentación, la implementación de diversas estrategias tales como la lectura, y el estudio en grupo. "Se aprende matemáticas haciendo matemáticas"

En lo que a la metodología respecta, los docentes en formación prefirien el trabajo en pequeños grupo, para el estudio y el aprendizaje de las matemáticas, ya que de esa forma tienen más seguridad en sí mismos, aunque son muchos los que expresan carecer de autoconfianza al resolver problemas matemáticos, en concreto el mismo porcentaje que experimenta inseguridad, desesperación y nerviosismo al atascarse o bloquearse siendo, sólo la mitad de ellos los que sienten calma y tranquilidad en dicho proceso de resolución.

$\mathrm{Al}$ interrogarles ¿Cómo cree Usted que los estudiantes aprenden matemáticas?, sus respuestas se centraron de manera general que ellos aprenden realizando ejercicios, que les permita posteriormente responder y llevar a práctica los diferentes conceptos. En este sentido el aprendizaje es visto como una copia "aprender es tener razones para justificar y explicara el éxito", que coincide con la visión platónica de las matemáticas de Ernest (1989). Otra la que le permita la aplicación de las reglas, teoremas y procedimientos matemáticos como un saber hacer, la concepción instrumental, muy propia de los docentes en ejercicio que orientan sus cursos en las ciencias básicas y las ingenierías, el cual parecen heredar los estudiantes, repercutiendo en un aprendizaje memorístico "profe deme la formula..." Y otra en la cual los estudiantes refieren que aprenden de la lectura y la reflexión permanente que le permita al estudiante ir construyendo sus propios conceptos; para posteriormente ser aplicados en cualquier contexto de acuerdo con la situación propuesta, en este sentido, el aprendizaje socio constructivo: "aprender es un proceso de construcción de conocimientos con 
la ayuda de un experto", visión de resolución de que los docentes en formación aparece como una buena oportunidad.

Los docentes en formación en sus clases prácticas de aula prefieren los métodos que les gustaban como estudiantes, enseñando de la misma forma en que fueron enseñados, tal como lo hacen los docentes en ejercicio que evocan a sus docentes, mostrando unas concepciones e imágenes pedagógicas muy estables y resistentes al cambio, fruto de su largo periodo de escolaridad, es decir, están influidos por la enseñanza que han visto y experimentado. Las distintas respuestas encontrados, pareciese que los docentes en formación estarán heredando las concepciones de los docentes en ejercicio, pues a lo largo de los discursos privilegian la manera como algunos de sus docentes orientan sus clases; clases que generalmente son tradicionales y donde impera la clase magistral y el rol protagónico del docente. Entre las estrategias que utilizan y recomiendan para aprender las matemáticas los docentes en formación a sus estudiantes las podemos clasificar en tres grandes grupos: Uno el de la disciplina, la cual implica dedicación, paciencia, atención y responsabilidad. Finalmente el de realizar una reflexión constante de cada uno de los conceptos matemáticos que le permitan al estudiante la construcción de sus propios conceptos; reflexión que se logra mediante el afianzamiento de diversos procesos que le permiten enfrentarse a determinada situación.

\section{Reflexiones y conclusiones}

Existe una poderosa influencia del contexto social, cuando se enseña y se aprenden las matemáticas Esto resulta de la expectativa de los demás, incluyendo los estudiantes, padres, colegas (otros profesores) y los superiores, así como del currículo institucionalizado: el texto adoptado o el esquema curricular, el sistema de evaluación, y en general el sistema nacional de escolaridad.
- La formación inicial de profesores se debe tener en cuenta los antecedentes escolares de los estudiantes, con la finalidad de analizar sus concepciones sobre las Matemáticas y su enseñanza-aprendizaje, y actuar de forma que éstos reflexionen sobre la naturaleza del contenido que aprenden e impartirán en un futuro y de su papel como alumnos y como futuros profesores.

- Los docentes en formación enseñan de la forma como les enseñan sus docentes, $y$ adquieren muchos de sus modelos, que seguramente forman parte de sus concepciones de la forma de ver su disciplina. Parece ser que de acuerdo con la concepción que tenga el docente acerca de las matemáticas, de esa misma manera enseña, trasladando muchos de sus elementos al estudiante.

- De acuerdo a los planteamientos de los sujetos participantes es evidente que es enseñando matemáticas como se aprende a enseñar matemáticas, pero, al mismo tiempo, enseñar matemáticas no es la única ni la mejor forma de aprender a enseñar matemáticas.

- La enseñanza de las matemáticas se caracterizó por el seguimiento de reglas y procedimientos, la práctica rutinaria de ejercicios, el uso de palabras clave y la falta de un contexto significativo para su aprendizaje., lo que coincide con lo afirmado por Moreno (2005)

- Los docentes en formación van forjando una idea de lo que son las matemáticas, de lo que significa hacer matemáticas y cómo transmitirla Esto propicia en el profesor una visión particular acerca de las matemáticas, la cual trata de trasmitir a sus alumnos a través de la enseñanza en el aula.

- Las concepciones que de los docentes en formación elaboran sobre las matemáticas, 
su papel en la enseñanza y los requerimientos para aprenderlas "dependen en gran medida" de los "mensajes que reciben" de sus profesores, lo cuales a su vez dependen de las concepciones de quienes los transmiten.

\section{Referencias}

Benítez, W. A., (2011) Concepciones acerca de las matemáticas, su enseñanza y aprendizaje: un estudio comparativo entre docentes en ejercicio y docentes en formación, Tesis de Maestría. Universidad del Cauca.

Ernest, P. (1989). The Impact of Beliefs on the Teaching of Mathematics at 6th International Congress of Mathematical Education, Budapest, August 1988. Budapest.

Moreno, M. (2005). El papel de la didáctica en la enseñanza del cálculo: evolución, estado actual y retos futuros. En S. Sociedad
Española de Investigación en Educación Matemática (Ed.), Noveno Simposio de la Sociedad Española de Educación Matemática SEIEM, (págs. 81-96). Córdoba- España.

Ponte, J. (1999). Las creencias y concepciones de maestros como un tema fundamental en formación de maestros [en línea], disponible en: http://www.educ.fc.ul.pt/docentes/ jponte/docs-sp/, recuperado: agosto 22 de 2012.

Remesal, A. (2006). Los problemas en la evaluación del aprendizaje matemático en la educación obligatoria:Perspectiva de profesores y alumnos. Tesis doctoral.

Thompson, A. (1992). Teacher's beliefs and conceptions: a synthesis of the research. New York: Macmillan.: En D.A. Grouws, (Ed.), Handbook on mathematics teaching and learning. 